\title{
Morphology of Ethylene/1-Octene Copolymers and Their Blends with High Density Polyethylene: A Microscopic Evaluation
}

\author{
Rameshwar Adhikari* \\ Central Department of Chemistry, Tribhuvan University, Kirtipur, Kathmandu, Nepal \\ E mail: nepalpolymer@yahoo.com
}

\begin{abstract}
The investigation into morphology formation in ethylene/l-octene copolymers (EOCs) comprising variable 1-octene content and their blends with high density polyethylene (HDPE) and hence their tensile mechanical properties have been reported. The morphological analysis by means of atomic force microscopy (AFM), transmission electron microscopy (TEM) and scanning electron microscopy (SEM) revealed the macrophase separation of the components in the blends. In contrast to well defined spherulitic morphology and lamellar structure of the HDPE, the EOCs exhibited progressively distorted lamellar morphology with increasing 1-octene content. At high 1-octene content, the EOC samples possessed the 'worm-like' crystals, which resemble the 'fringed micelles' discussed in the literature. The blends allow a balance of mechanical properties (stiffness and toughness) over a wide range as shown by tensile stress strain behavior of the blends.
\end{abstract}

Keywords: Atomic Force Microscopy (AFM), Electron Microscopy, Polyethylene, Ethylene/1-Octene Copolymer, Mechanical Properties

\section{Introduction}

The recent trend towards the development of polymers with tailored mechanical properties is to heterogenize the existing polymers and control their heterophase morphology via blending, copolymerisation and optimizing processing conditions. The block copolymerisation of glassy polymers (e.g., polystyrene) with dienes (e.g., polybutadiene or polyisoprene) has been successfully used to tailor the mechanical properties ranging from hard impact modified products to the elastomeric ones ${ }^{1}$. In spite of attractive properties, however, due to higher manufacturing costs, the block copolymers do not fulfil the demands of customers in the polymer market. Hence, there is still a need of strategy for the technical production of materials having desired properties profile on the basis of commodity plastics. In this regard, the copolymerisation of polyethylene with a $\alpha$-olefins has been shown to drastically alter the mechanical properties of the polyethylene at hand ${ }^{2,3}$. The use of single-site metallocene catalysts leads to the formation of homogeneous ethylene/ $\alpha$-olefin copolymers with uniform distribution of commoners and a narrow molar mass distribution ${ }^{2}$. In general, it is known that via copolymerisation with higher $\alpha$-olefin comonomers, the density and hence the melt flow index (MFI) of these copolymers may be adjusted in a controlled manner. Consequently, such copolymers have opened new avenues of producing polymers with tailored mechanical properties.

\section{* Corresponding author}


Unlike a crystallizable homopolymers (such as polyethylene, polypropylene, polyethylene oxide etc.), a copolymer is fragmented into a number of sequences by the occurrence of branching along the main chain backbone ${ }^{4}$. For instance, in polyethylene, the branches are almost always excluded from the crystal structure to be formed. As a result, the copolymers display a wide range of sequence lengths that can crystallize over a correspondingly wide range of temperatures. This feature forms the basis of most of the differences observed in the crystallization behaviour between the homo- and copolymers ${ }^{4}$. The homopolymers crystallization is largely influenced by the extent of chain entanglement in the melt, the chain length and its distribution, and the under-cooling. However, this phenomenon in copolymers is predominantly influenced by the degree of branching which controls the length of crystallizable sequences and their distribution. In polyethylene, the branching characteristics can be governed by the nature of the catalyst used ${ }^{4-7}$.

Earlier studies reported on the structure, morphology, crystallisation and melting behaviour of these new types of copolymers and blends and their correlation with the tensile properties ${ }^{5}$. Owing to their significantly better mechanical and thermal properties, rapidly increasing industrial applications of metallocene based ethylene copolymers have been reported ${ }^{6}$. During the recent years, a lot of progress has been made in the structural characterization, their chemical modification ${ }^{8-12}$, blending with different polymers of these kinds of polymers.

The aim of present work is to analyze the phase morphology of selected ethylene/1-octene copolymers (EOCs) and their blends with high density polyethylene (HDPE) correlating these structures with their tensile mechanical behaviour.

\section{Experimental Methods}

\section{Materials and Sample Preparation}

Two different types of ethylene/1-octene copolymer (named as EOC1, EOC2 and EOC3) synthesized by the INSITE ${ }^{\mathrm{TM}}$ technology and one HDPE, products of Dow Chemical Company, were used in the study. The comonomer 1-octene in the copolymers lies in the range of 0-35 wt.- $\%$. A twin-screw extruder was used to prepare the binary HDPE/EOC3 blends by melt mixing. Approximately $1 \mathrm{~mm}$ thick plaques of each sample were compression molded at $160{ }^{\circ} \mathrm{C}$ and subsequent cooling under pressure. The characteristics of samples used to prepare the blends are presented in Table 1.

Table 1. Some of the important characteristics of the samples used in this study

\begin{tabular}{llccc}
\hline Sample code & Commercial name & 1-octene wt.-\% & density $\left(\mathrm{g} / \mathrm{cm}^{3}\right)$ & melting point $\left({ }^{\circ} \mathrm{C}\right)$ \\
\hline HDPE & HDPE 53050 E & 0 & 0.953 & 131 \\
EOC2 & Affinity PL1850 & 17.2 & 0.902 & 101 \\
EOC3 & Affinity EG8150 & 35.0 & 0.868 & 55 \\
\hline
\end{tabular}

\section{Characterization Methods}

The samples were characterized by different microscopic techniques (viz. atomic force microscopy, transmission electron microscopy, and scanning electron microscopy) and tensile testing.

Transmission electron microscopy (TEM) was performed on thin sections, approximately $70 \mathrm{~nm}$ thick, and stained with ruthenium tetroxide vapor using a $200 \mathrm{kV}$ TEM (Jeol, Japan). The thin sectioning was performed with the aid of an Ultramicrotome (Leica, Austria) equipped with a diamond knife (Diatome, Switzerland) operated at $-100{ }^{\circ} \mathrm{C}$.

$$
-6 \text { - }
$$


Atomic Force Microscopy (AFM) was performed using a Digital Instruments, Multimode atomic force microscope equipped with Nanoscope IIIa controller in tapping mode to image the phase morphology of the samples. As specimens, the block of the samples left behind after cryo-ultramicrotomy for the TEM was used.

Scanning electron microscopy (JSM 6300, JEOL) was used to visualize the structural details of the bulk samples. The surfaces prepared by microtomy at subjected to permanganic etching and later sputtered coated with thin gold film to avoid charging and irradiation damage during the SEM observations.

Tensile testing was performed using a universal tensile machine (Zwick 1425) at $23{ }^{\circ} \mathrm{C}$ to characterize the mechanical properties according to the DIN 53504 norms.

\section{Results and Discussion}

\section{Morphology of EOCs and HDPE/EOC3 Blends}

It is well known that high density polyethylene (HDPE) is a semicrystalline polymer. Fig. 1 depicts the AFM phase images of different magnifications showing the typical morphology of HDPE. In those phase images and the images to follow later in this articles, the brighter areas will correspond to relatively stiffer or harder regions (for instance, the crystalline regions) and the darker one the softer regions (for instance, the amorphous regions) of the polymer. In agreement with earlier results obtained by transmission electron microscopy, a banded spherulitic structure is observed. A parallel array of crystalline lamellar bundles is found to from the spherulitic centre, each crystalline lamella being clearly separated by amorphous inter-crystalline amorphous layers.
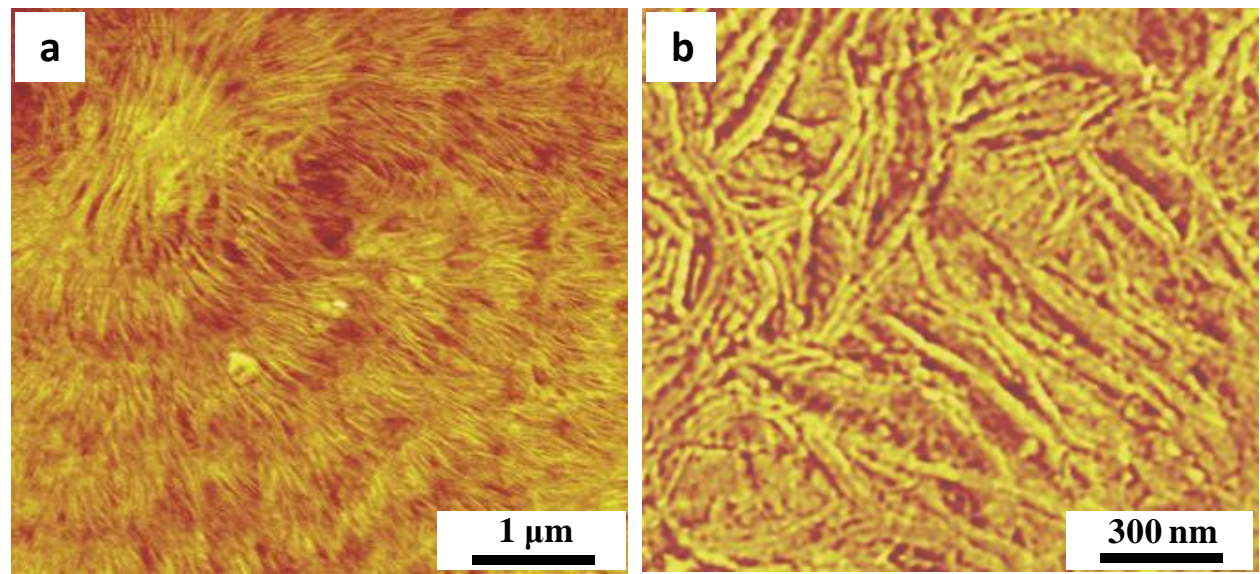

Figure 1. AFM phase images of HDPE with different magnifications.

On copolymerizing ethylene monomers with 1-octene using newly developed metallocene catalysts, it has been shown that the comonomers can be added to produce a homogeneously distributed C-6 side chains in the main polyethylene chain ${ }^{2}$. This affects, as shown by microscopic and calorimetric investigations, greatly the crystallization behavior and morphology formation ${ }^{4,8}$.

The SFM phase micrographs of the ethylene/1-octene copolymer at different comonomer content are presented in Fig. 2. When the 1-octene content is $17.5 \%$, the clear lamellar structure gets distorted (see Fig. 2a) and at higher 1-octene content, a dramatic change in the morphology occurs (see Fig. 2b). Thus, with increasing 1-octene content, the spherulitic structure gradually disappears and the lamellae structure 
becomes progressively destroyed. At higher 1-octene content, worm-like crystals are observed. The crystals resemble the fringed micelles discussed in the literature. The structural changes in the ethylene copolymers with increasing comonomer content as elucidated by the SFM results are in line with a change in their density, crystallinity and dynamic mechanical behavior. For example, a strong change in density and melt flow index at higher 1-octene content was observed ${ }^{7}$ (see also Table 1).


Figure 2. AFM phase images showing morphology of: a) EOC2 (17.5 wt.-\% 1-octene) and b) EOC3 (25 wt. $-\%$ 1-octene).

The effect of morphological changes in polyethylene provoked by the copolymerization with $\alpha$ olefin (such as 1-octene) can be observed in the blends of HDPE with the copolymer and even in the blends of the copolymers. In this paper, the morphology of the blends of HDPE and EOC will be evaluated on the basis of AFM and TEM results. Fig. 3 depicts the tapping mode AFM phase images of a blend with HDPE/EOC3 weight ratio of 60/40. As the specimen of the AFM studies was prepared by ultramicrotomy of the bulk sample, the micrographs illustrate the true internal morphology of the respective sample.

The micrographs of a bulk blend (60\% HDPE and 40\% EOC3) given in Fig. 3 clearly show two kinds of areas: darker areas representing seemingly predominant amorphous phase and the bright stripes of long needles depicting the crystalline domains. Thus, the micrographs clearly demonstrate the phase separation between the HDPE and EOC3 components. The HDPE phase appears in the form of long needle-like crystals of different lengths. Occasionally, the crystalline domains form the woven structures in contrast to the highly organized parallel array of lamellae of the parent HDPE. The brighter areas in contrast, on careful examinations (see Fig. 3b), reveal the structure similar to that found in Fig. 2b, i.e. of EOC3.

The results suggests that the blend components undergo phase-segregation in the blends whereby the organization of the lamellar crystals is greatly influenced in the respect that they grow into long and less intact needle-like forms.

The results obtained by AFM are complemented by TEM results presented in Fig. 4. The selective staining of the amorphous phase by ruthenium tetroxide makes the highly dense crystalline domains appear bright in the TEM image. Obviously, the darker phase is the amorphous inter-lamellar region or the EOC3-rich region. The EOC3-rich region further reveals the tiny worm-like domains dispersed in an amorphous matrix in agreement of SFM results discussed earlier. The TEM picture in Fig. 4 illustrates that the EOC3 phase lacks the well defined lamellar crystals. Furthermore, formation of needle-like 
crystals without the evidence of well developed spherulitic texture in the blends, especially at higher EOC 3 content, was noticed, which indicate that the crystallization process of the HDPE is suppressed in the mixtures.
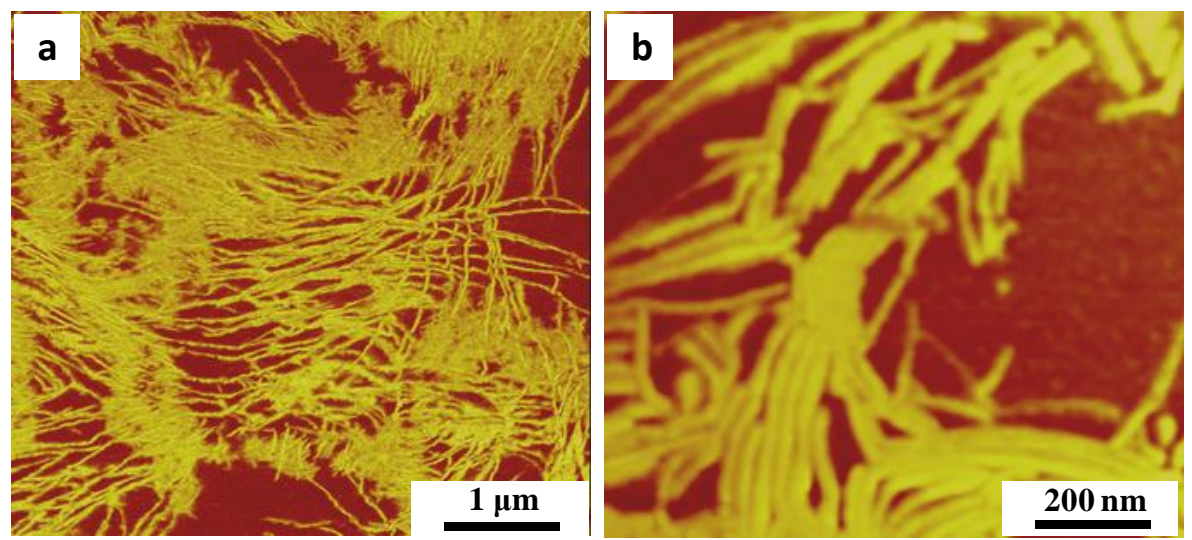

Figure 3. AFM phase images of different magnifications showing morphology of a HDPE/EOC3 (60/40) blend; brighter and darker regions correspond to HDPE and EOC3 phases, respectively.
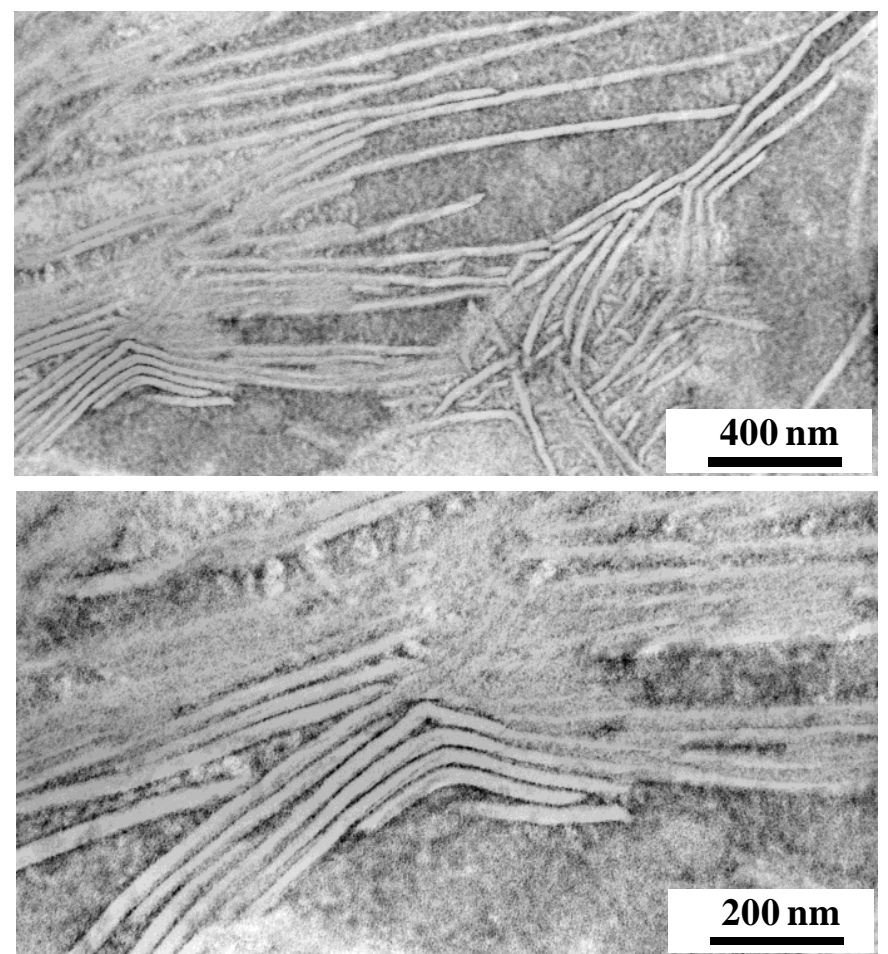

Figure 4. TEM images of different magnifications showing the morphology of a HDPE/EOC (60/40) blend; brighter and darker regions correspond to HDPE and EOC3 phases, respectively. 


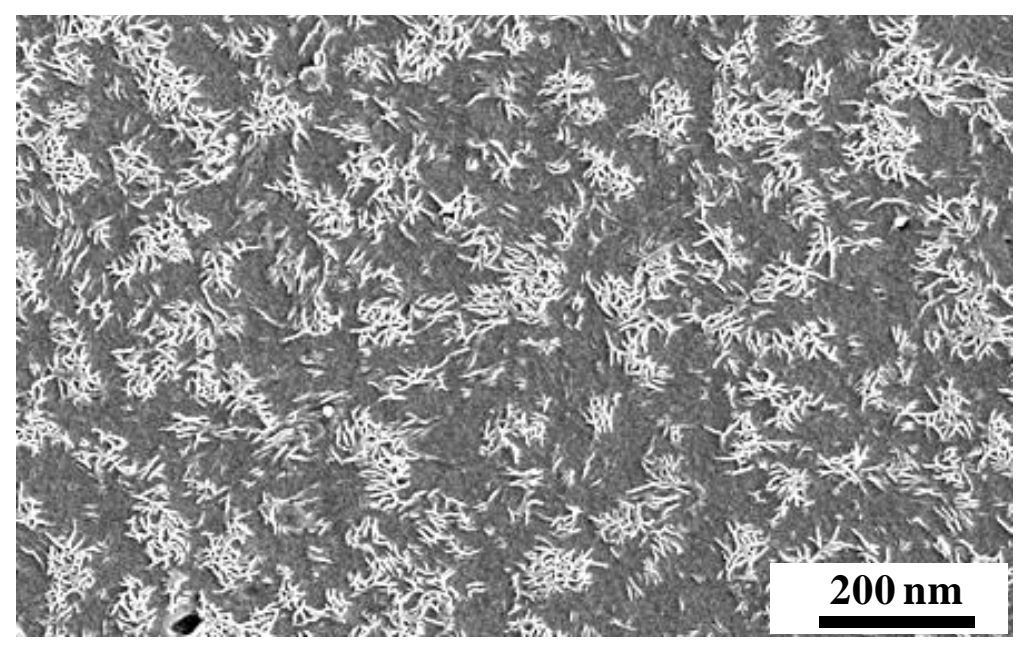

Figure 5. SEM image showing the morphology of a HDPE/EOC (25/75) blend; brighter and darker regions correspond to HDPE and EOC3 phases, respectively.

Furthermore, the blends of HDPE with copolymers showed two the melting endotherms. The melting points corresponded to that of the pure blend components indicating that the two components crystallized as separate crystalline phases in all the blends. Thus, melting and crystallization behavior of the blends suggested that there is no co-crystallization ${ }^{12}$.

The segregation of the HDPE and EOC3 phases becomes more and more pronounced in the blends when the fraction of EOC3 is higher. The phenomenon is illustrated by investigating a blend comprising 25 parts by weight of HDPE and 75 parts by weight of EOC3. The blend morphology was imaged by SEM after the permanganic etching of the specimen in order to expose the crystalline domains by removing the amorphous and loosely bound componets. The results are presented in Fig. 5. In the micrographs, bright spots represent the more rigid form of the polymer blend which was stable against permanganic etchant attack. Thus, the darker areas in the micrographs represent the loosely bound regions of the blend or more amorphous part of the polymer (i.e. the EOC3-rich phase).

A closer look into the micrograph reveal that the crystalline regions of the blend comprises the bright thread-like structures, which are several tens of nanometers thick, and thus represents the lamellar bundles. Limited by the resolution power of the SEM, the individual lamellae cannot be recognized, however, in Fig. 5. The SEM results again attest the segregation of the blend components into macroscopic domains in conformity with the AFM and TEM results.

\section{Mechanical Behavior}

Fig. 6 compares the tensile stress-strain behavior of HDPE with the EOCs and some HDPE/EOC3 blends. As required by the change in morphology, the mechanical properties change from plastic to elastomeric behavior with increasing 1-octene content.

The deformation of HDPE is accompanied by a well defined yield point (yield stress $=26 \mathrm{MPa}$ ), undergoes large plastic deformation (cold drawing) and strain hardening behavior. Ultimately, the stress level reaches approximately $40 \mathrm{MPa}$ when the sample undergoes fracture at an elongation of about $550 \%$. (Curve I, in Fig. 6).

When $17.2 \%$ of 1 -octene is present in the copolymer, the tensile properties change significantly (see Curve II, in Fig. 6): the yield stress is much lower than that of HDPE, there are two diffuse yield points (similar to that observed in low density polyethylene grades), and slight improvement in elongation at 
break at the cost of strength. At higher 1-octene content (e.g., EOC3, Curve III in Fig. 6), the copolymer behaves as a thermoplastic elastomer, where the micellar crystals may act as physical cross-links at room temperature. For the HDPE/EOC3 blends, it can be seen that, depending on the blend composition, mechanical behavior is intermediate between that of HDPE and EOC3. Thus, the properties of the blends can be controlled by blending them, allowing a balance of stiffness and toughness over a wide range.

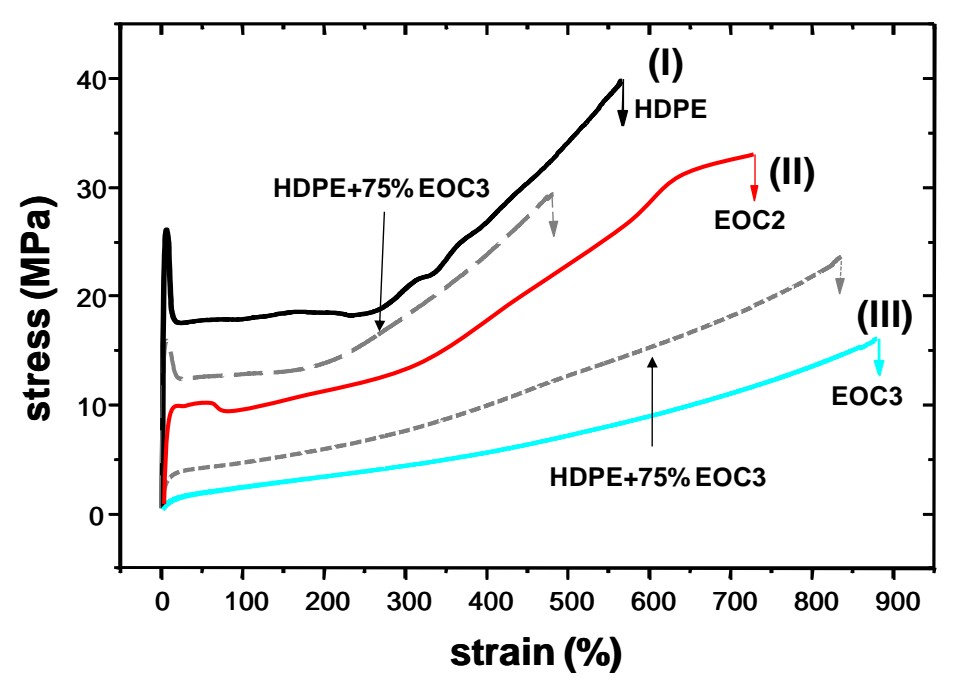

Figure 6. Stress-strain curves of EOCs and some HDPE/EOC3 blends recorded at $23{ }^{\circ} \mathrm{C}$.

\section{Conclusions}

The morphological features of selected ethylene/1-octene copolymers (EOCs) and their blends with high density polyethylene (HDPE) were studied by different microscopic techniques. It has been found that, at high 1-octene content, the EOC samples reveal the worm-like crystals, which resemble the fringed micelles discussed in the literature. It was found that, the components in HDPE/EOC blends possess a strong tendency towards segregation. Higher the amount of 1-octene in given EOC, stronger is the inclination towards phase separation. The multi-component polyolefins studied showed, depending on the comonomer content, a variety of crystalline structures ranging from lamellae to micellar fragments. Consequently, it was possible to tailor their mechanical properties over a wide range.

\section{Acknowledgements}

The author acknowledges research group of Prof. Goerg H. Michler (Martin Luther University HalleWittenberg, Halle/Saale, Germany), in particular Dr. Reinhold, Mr. W. Lebek and Ms. Sylvia Goerlitz, for providing the laboratory facilities to conduct the AFM and TEM experiments.

\section{References}

1. G. Holden, "Understanding Thermoplastic Elastomers", Hanser Verlag Munich 2000.

2. S. Bensason, S. Nazarenko, S. Chum, A. Hiltner, E. Baer, Polymer, 1997, 38, 3513. 
3. R. Godehardt, S. Rudolf, W. Lebek, S. Goerlitz, R. Adhikari, E. Allert, J. Giesemann and G. H. Michler, J. Macromol. Sci. B, 1999, 38, 817.

4. C. Subramaniam, Morphology, Crystallization and Melting Behaviors of Random Copolymers of Ethylene with 1-Butene, 1-Pentene and 1-Hexene, Ph. D. Thesis, Virginia Polytechnic Institute \& State University, 1999.

5. S. Bensason, J. Minick, A. Moet, S. Chum, A. Hiltner, E. Baer, J. Polym. Sci. Part B: Polym. Phys. 1996, 34, 1301.

6. R. Adhikari, R. Godehardt, W. Lebek, S. Frangov, G. Michler, H. Radusch, F. J. Balta' Calleja, Polym. Adv. Tech., 2005, 16, 156.

7. S. Frangov, Morphologie und Deformationsverhalten von Blends aus Polyethylen hoher Dichte und Ethylen-1-Octen-Copolymeren, Shaker Verlag GmbH, Aachen, 2005.

8. M. U. Wahit, A. Hassan, Z. A. Mohd Ishak, T. Czigány, eXPRESS, Polym. Lett. 2009, 3, 309.

9. A. Biswas, A. Bandyopadhyay, N. K. Singha, A. K. Bhowmick, J. Appl. Polym. Sci., 2009, 114, 3906.

10. A. Biswas, A. Bandyopadhyay, N. K. Singha, A. K. Bhowmick, J. Mater. Sci. 2009,44, 3125.

11. F. G. Karssenberg, Chain Microstructures of Homogeneous Olefin Copolymers and Characteristics of Single Site Catalysts, Technische Universiteit Eindhoven, 2005.

12. P. Svoboda, S. Poongavalappil, R. Theravalappil,D. Svobodova, P. Mokrejs, K. Kolomaznik, T. Ougizawa, T. Inoue, J. Appl. Polym. Sci., 2011, 121, 521. 